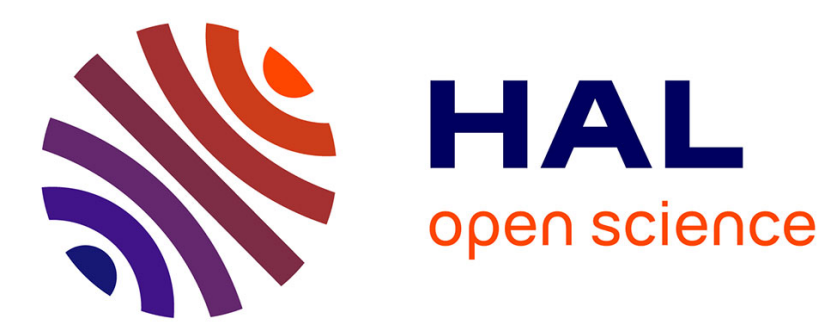

\title{
Relation between unit operators proximity and their associated spectral measures
}

\author{
Alain Boudou, Sylvie Viguier-Pla
}

\section{To cite this version:}

Alain Boudou, Sylvie Viguier-Pla. Relation between unit operators proximity and their associated spectral measures. Statistics and Probability Letters, 2010, 10.1016/j.spl.2010.07.016 . hal-00691783

\section{HAL Id: hal-00691783 \\ https://hal.science/hal-00691783}

Submitted on 27 Apr 2012

HAL is a multi-disciplinary open access archive for the deposit and dissemination of scientific research documents, whether they are published or not. The documents may come from teaching and research institutions in France or abroad, or from public or private research centers.
L'archive ouverte pluridisciplinaire HAL, est destinée au dépôt et à la diffusion de documents scientifiques de niveau recherche, publiés ou non, émanant des établissements d'enseignement et de recherche français ou étrangers, des laboratoires publics ou privés. 


\section{Accepted Manuscript}

Relation between unit operators proximity and their associated spectral

measures

Alain Boudou, Sylvie Viguier-pla

PII:

$$
\text { S0167-7152(10)00210-5 }
$$

DOI:

$$
\text { 10.1016/j.spl.2010.07.016 }
$$

Reference: $\quad$ STAPRO 5754

To appear in: Statistics and Probability Letters

Received date: 7 January 2010

Revised date: 19 July 2010

Accepted date: 21 July 2010

Please cite this article as: Boudou, A., Viguier-pla, S., Relation between unit operators proximity and their associated spectral measures. Statistics and Probability Letters (2010), doi:10.1016/j.spl.2010.07.016

This is a PDF file of an unedited manuscript that has been accepted for publication. As a service to our customers we are providing this early version of the manuscript. The manuscript will undergo copyediting, typesetting, and review of the resulting proof before it is published in its final form. Please note that during the production process errors may be discovered which could affect the content, and all legal disclaimers that apply to the journal pertain. 


\title{
RELATION BETWEEN UNIT OPERATORS PROXIMITY AND THEIR ASSOCIATED
} SPECTRAL MEASURES

\author{
Alain BOUDOU and Sylvie VIGUIER-PLA ${ }^{1}$ \\ Equipe de Stat. et Proba., Institut de Mathématiques, UMR5219, \\ Université Paul Sabatier, 118 Route de Narbonne, F-31062 Toulouse Cedex 9, France \\ boudou@math.univ-toulouse.fr, viguier@math.univ-toulouse.fr
}

Abstract. In this paper, we study how the locally concentration of the spectral measure expresses in the temporal domain for stationary processes. For this purpose, we establish an equivalence between the proximity of the shift operators (which are unit operators) and the associated projector-valued spectral measures. An illustration is given.

Keywords. Random measures, Stationnary processes, Fourier transform, Stochastic integrals, Spectral measures

AMS subject classification. 60G57, 60G10,60B15, 60H05

\section{Introduction}

Every unit operator can be univoquely associated with a projector valued spectral measure (cf. Riesz and Nagy, 1991). Therefore, when $P$ is an orthogonal projector, $U=(I-P)+e^{i \lambda} P$ is a unit operator associated with the spectral measure $\delta_{0}().(I-P)+\delta_{\lambda}() P.\left(\delta_{0}\right.$ and $\delta_{\lambda}$ are the Dirac measures at 0 and $\left.\lambda\right)$. In the same way, the unit operator $I$ is associated with the spectral measure $\delta_{0}()$.$I .$

We naturally think that when two unit operators $U$ and $U^{\prime}$ are close together, the same thing happens for their associated spectral measures $\mathcal{E}$ and $\mathcal{E}^{\prime}$, in a way to be defined.

The proximity between the operators $U$ and $U^{\prime}$ is evaluated by the well known norm $\left\|U-U^{\prime}\right\|_{\mathcal{L}}=\sup \{\| U(X)-$ $\left.U^{\prime}(X)\|;\| X \|=1\right\}$. In order to evaluate the neighborhood between the spectral measures $\mathcal{E}$ and $\mathcal{E}^{\prime}$ we could consider the quantity $\sup \left\{\left\|\mathcal{E}(A)-\mathcal{E}^{\prime}(A)\right\|_{\mathcal{L}} ; A \in \mathcal{B}\right\}$. However, such a choice is not appropriate because, for example, when $U=(I-P)+e^{i \lambda} P$ and $U^{\prime}=I$, we get $\left\|U-U^{\prime}\right\|_{\mathcal{L}} \leq|\lambda|$ and $\sup \left\{\left\|\mathcal{E}(A)-\mathcal{E}^{\prime}(A)\right\|_{\mathcal{L}} ; A \in \mathcal{B}\right\}=1$. This means that the unit operators $U$ and $U^{\prime}$ can be very close, when $\lambda$ is small, but the quantity $\sup \left\{\left\|\mathcal{E}(A)-\mathcal{E}^{\prime}(A)\right\|_{\mathcal{L}} ; A \in \mathcal{B}\right\}$, equal to 1 , do not translate, in any way, the proximity between the spectral measures.

In paragraph 2, in order to express the neighborhood between two spectral measures, we introduce the notion of $\alpha$-equivalence of spectral measures, notion based on a well known relation of partial order between projectors.

In the following paragraph, we show that the proximity between unit operators, when they commute, implies that of associated spectral measures from an $\alpha$-equivalence point of view. Conversely, the $\alpha$-equivalence of spectral measures which commute implies proximity of the corresponding unit operators. Therefore, it is clear that the notion of $\alpha-$ equivalence in the frequency domain restitutes correctly the proximity between unit operators.

This way of studying proximity is different from what we can find usually (Kato, 1980), because the type of perturbation is not parametrized. An application of this study could be the study of quasi-periodicity, following the ideas of Dehay (2007).

Then we have a tool to deal with problems of perturbation concerning unit operators. This aspect is important in the study of stationary processes, since shift operators (Brillinger, 2001, Bosq, 2000, Rozanov, 1967) are unit operators. Thanks to the previous results we can prove that if the unit operator $U: X_{n} \longmapsto X_{n+1}$, associated with a stationary series $\left(X_{n}\right)_{n \in \mathbb{Z}}$, is such that $U^{k}$ is close to $I$, and so, $X_{k+n}$ close to $X_{n}$, then the random measure $Z$, whose Fourier transform is $\left(X_{n}\right)_{n \in \mathbb{Z}}$, is concentrated into the neighborhood of the points $\lambda_{q}$ such that $\left(e^{i \lambda_{q}}\right)^{k}=1$. Conversely, if $Z$ is concentrated in the neighborhood of such points of the spectrum, then the shift operator $U$ associated with the stationary series $\left(\int e^{i . n} d Z\right)_{n \in \mathbb{Z}}$ is such that $U^{k}$ is close to $I$. This result illustrates the duality between the temporal and the frequencial points of vue. We obtain similar results for stationary random functions.

\section{Notation and preliminary results}

This paragraph is devoted to recall the necessary tools for the understanding of the text, to precise notation and to establish some preliminary results.

We denote by $H$ a separable $\mathbb{C}$-Hilbert space and $\mathcal{P}(H)$ the set of its orthogonal projectors. Of course, when $H$ is $L^{2}(\Omega, \mathcal{A}, P)$ or $L_{\mathbb{C}^{p}}^{2}(\Omega, \mathcal{A}, P)$, this study becomes part of the studies of stochastic processes.

\footnotetext{
${ }^{1}$ Corresponding author : viguier@math.univ-toulouse.fr
} 


\subsection{The topologic group $\Pi$}

The set $\Pi=\left[-\pi ; \pi\left[\right.\right.$ is a compact commutative group for the binary operation $\oplus:\left(\lambda_{1}, \lambda_{2}\right) \in \Pi \times \Pi \longmapsto \lambda_{1}+\lambda_{2}-$ $2 \pi\left[\frac{\lambda_{1}+\lambda_{2}+\pi}{2 \pi}\right] \in \Pi([x]$ is the integer part of $x)$ and for the distance $d:\left(\lambda_{1}, \lambda_{2}\right) \in \Pi \times \Pi \longmapsto\left|\lambda_{1} \ominus \lambda_{2}\right| \in \mathbb{R}_{+}$, which is invariant by translation. This last one induces a topology, for which the applications $w: \lambda \in \Pi \longmapsto \ominus \lambda \in \Pi$ and $S:\left(\lambda_{1}, \lambda_{2}\right) \in \Pi \times \Pi \longmapsto \lambda_{1} \oplus \lambda_{2} \in \Pi$ are continuous (where, of course, $\ominus \lambda$ denotes the symmetric of $\lambda$ ). The family of compact (or closed) subsets for this topology is denoted $\mathcal{K}$, and $\mathcal{B}$ denotes the family of borelians.

For all $(A, \lambda)$ of $\mathcal{B} \times \Pi$, the set $A \oplus \lambda$ is the reciprocal image of $A$ by the application $\lambda^{\prime} \in \Pi \longmapsto \lambda^{\prime} \ominus \lambda \in \Pi$ which is, from the properties of the topologic group, continuous. We can then give the

Lemma 1.1 For all $\lambda$ of $\Pi$, when $A$ is an element of $\mathcal{B}$ (resp. an open subset of $\Pi$; a closed subset of $\Pi$ ), then $A \oplus \lambda$ is an element of $\mathcal{B}$ (resp. an open subset of $\Pi$; a closed subset of $\Pi$ ).

For each couple $(A, B)$ of subsets of $\Pi$, we denote by $A \oplus B$ the set of the elements of $\Pi$ that can be writen $\lambda_{A} \oplus \lambda_{B}$, where $\left(\lambda_{A}, \lambda_{B}\right)$ belongs to $A \times B$. Let examine the

Lemma 1.2 For all $\alpha$ of $[0, \pi[$, when $B$ is an element of $\mathcal{B}$, the same happens for $B \oplus[-\alpha, \alpha]$.

Proof. The result is obvious when $\alpha=0$. When $\alpha$ is different from 0 , it is the consequence of $B \oplus[-\alpha, \alpha]=(B \oplus]-\alpha, \alpha[) \cup(B \oplus(\ominus \alpha)) \cup(B \oplus \alpha)=\left(\cup_{\lambda \in B}(\lambda \oplus]-\alpha, \alpha[)\right) \cup(B \oplus(\ominus \alpha)) \cup(B \oplus \alpha)$.

In the particular case where $B$ is compact, from $S(B \times[-\alpha, \alpha])=B \oplus[-\alpha, \alpha]$, the previous result becomes

Lemma 1.3 For all $\alpha$ of $[0, \pi[$, when $B$ is a compact subset of $\Pi$, the same happens for $B \oplus[-\alpha, \alpha]$.

Let end this section by a result that will be used in paragraph 2 .

Lemma 1.4 If $\left(\alpha_{n}\right)_{n \in \mathbb{N}}$ is a decreasing sequence of elements of $[0, \pi[$ which converges towards $\alpha$, then, for all $B$ of $\mathcal{K}$, we have $B \oplus[-\alpha, \alpha]=\cap_{n \in \mathbb{N}}\left(B \oplus\left[-\alpha_{n}, \alpha_{n}\right]\right)$.

Proof. Let $\gamma$ be an element of $\cap_{n \in \mathbb{N}}\left(B \oplus\left[-\alpha_{n}, \alpha_{n}\right]\right)$. With every $n$ of $\mathbb{N}$ we can associate an element $\left(\lambda_{n}, \lambda_{n}^{\prime}\right)$ of $B \times\left[-\alpha_{n}, \alpha_{n}\right]$ such that $\gamma=\lambda_{n} \oplus \lambda_{n}^{\prime}$. The compacity of $\Pi$ authorizes to say that a convergent sub-sequence $\left(\lambda_{n_{k}}\right)_{k \in \mathbb{N}}$, of $\left(\lambda_{n}\right)_{n \in \mathbb{N}}$ exists. Its limit $\lambda$ belongs to $B$. We then establish that $\gamma \ominus \lambda$ is the limit of the sequence $\left(\lambda_{n_{k}}^{\prime}\right)_{n \in \mathbb{N}}$. From the relations $\left|\lambda_{n_{k}}^{\prime}\right| \leq \alpha_{n_{k}}$ and $\lim _{k} \alpha_{n_{k}}=\alpha$, we deduce that $\gamma \ominus \lambda \in[-\alpha, \alpha]$, and $\gamma=\lambda \oplus(\gamma \ominus \lambda)$ belongs to $B \oplus[-\alpha, \alpha]$. As besides, $(B \oplus[-\alpha, \alpha]) \subset\left(\cap_{n \in \mathbb{N}}\left(B \oplus\left[-\alpha_{n}, \alpha_{n}\right]\right)\right)$, the expected equality stands.

\subsection{Random measures, stationary functions}

In this section, we shortly recall the well-known biunivoque correspondence between a random measure and a stationary continuous random function.

Let $G$ be a locally compact abelian group whose dual $\widehat{G}$ admits a countable basis. So $G$ may be any of the sets $\mathbb{Z}, \mathbb{Z}^{k}$, $\mathbb{R}, \mathbb{R}^{k}, \Pi$ or $\Pi^{k}$

A stationary continuous random function (c.r.f.) $\left(X_{g}\right)_{g \in G}$, defined on $G$ and with values in $H$, is a family of elements of $H$ such that $\left.\left\langle X_{g}, X_{g^{\prime}}\right\rangle=<X_{g-g^{\prime}}, X_{0}\right\rangle$, for all couple $\left(g, g^{\prime}\right)$ of elements of $G$, and such that the application $g \in G \longmapsto X_{g} \in H$ is continuous. When $G=\mathbb{Z}$ we talk about stationary series.

A random measure (r.m.) $Z$ is a vector measure defined on $\mathcal{B}_{\widehat{G}}$, with values in $H$ such that $\langle Z(A), Z(B)\rangle=0$, for all couple $(A, B)$ of disjoint elements of $\mathcal{B}_{\widehat{G}}$.

The application $\mu_{Z}: A \in \mathcal{B}_{\widehat{G}} \longmapsto\|Z(A)\|^{2} \in \mathbb{R}^{+}$is obviously a bounded measure. The stochastic integral, with respect to $Z$, can be defined as the unique isometry from $L^{2}\left(\widehat{G}, \mathcal{B}_{\widehat{G}}, \mu_{Z}\right)$ onto $H_{Z}=\overline{\text { vect }}\left\{Z(A) ; A \in \mathcal{B}_{\widehat{G}}\right\}$ which associates $Z(A)\left(=\int 1_{A} d Z\right)$ with $1_{A}$, for all $A$ of $\mathcal{B}_{\widehat{G}}$.

The family $\left(\int(., g)_{\widehat{G} G} d Z\right)_{g \in G}$ is a stationary c.r.f.. Conversely, with each stationary c.r.f. $\left(X_{g}\right)_{g \in G}$, we can associate one and only one r.m. $Z$, called r.m. associated with the stationary c.r.f. $\left(X_{g}\right)_{g \in G}$, such that $\int(., g)_{\widehat{G} G} d Z=X_{g}$, for all $g$ of $G$.

If $\varphi$ is an element of $L^{2}\left(\widehat{G}, \mathcal{B}_{\widehat{G}}, \mu_{Z}\right),\left(\int(., g)_{\widehat{G} G} \varphi(.) d Z\right)_{g \in G}$ is a stationary c.r.f., called image of $\left(X_{g}\right)_{g \in G}$ by the filter $\varphi$; its associated r.m. is the application $Z_{\varphi}: A \in \mathcal{B}_{\widehat{G}} \longmapsto \int 1_{A} \varphi d Z \in \mathcal{B}_{\widehat{G}}$.

In the particular case where $G=\mathbb{Z}$ (resp. $G=\mathbb{R}$ ) the dual group is identified with $\Pi$ (resp. $\mathbb{R})$, the r.m. $Z$ associated with a stationary series $\left(X_{n}\right)_{n \in \mathbb{Z}}$ (resp. with a stationary c.r.f. $\left.\left(X_{t}\right)_{t \in \mathbb{R}}\right)$ is defined on $\mathcal{B}$ (resp. $\mathcal{B}_{\mathbb{R}}$ the Borel $\sigma$-field of $\mathbb{R}$ ), for all $n$ of $\mathbb{Z}$ (resp. for all $t$ of $\mathbb{R}$ ) we have $\int e^{i . n} d Z=X_{n}\left(\right.$ resp. $\left.\int e^{i . t} d Z=X_{t}\right)$.

We can show that to r.m.'s defined on $\mathcal{B}$ that match on $\mathcal{K}$ are equal. 


\section{$1.3 \quad$ Spectral measures}

In this section, we define the spectral measure and associated various notions, as the convolution product.

Let $(T, \mathcal{T})$ and $\left(T^{\prime}, \mathcal{T}^{\prime}\right)$ be measurable spaces.

A spectral measure (s.m.) $\mathcal{E}$ on $\mathcal{T}$ for $H$ is an application from $\mathcal{T}$ onto $\mathcal{P}(H)$ such that:

i) $\mathcal{E}\left(A_{1} \cup A_{2}\right)=\mathcal{E}\left(A_{1}\right)+\mathcal{E}\left(A_{2}\right)$, for all couple $\left(A_{1}, A_{2}\right)$ of disjoint elements of $\mathcal{T}$;

ii) $\mathcal{E}(T)=I_{H}$;

iii) $\lim _{n} \mathcal{E}\left(A_{n}\right)(X)=0$ for each decreasing sequence $\left(A_{n}\right)_{n \in \mathbb{N}}$ of elements of $\mathcal{T}$ converging to $\emptyset$ and for all $X$ of $H$. Hence, it can be shown that, for all couple $(A, B)$ of elements of $\mathcal{T}, \mathcal{E}(A) \circ \mathcal{E}(B)=\mathcal{E}(A \cap B)$, and that $\mathcal{E}(\emptyset)=0$. For all $X$ of $H$, the application $Z_{\mathcal{E}}^{X}: A \in \mathcal{T} \longmapsto \mathcal{E}(A) X \in H$ is a r.m..

We also can establish that $\lim _{n} \mathcal{E}\left(A_{n}\right) X=\mathcal{E}\left(\cap_{n \in \mathbb{N}} A_{n}\right) X$, when $\left(A_{n}\right)_{n \in \mathbb{N}}$ is a decreasing sequence of elements of $\mathcal{T}$.

When $f$ is a measurable application from $T$ to $T^{\prime}$, the application $f(\mathcal{E}): A^{\prime} \in \mathcal{T}^{\prime} \longmapsto \mathcal{E}\left(f^{-1} A^{\prime}\right) \in \mathcal{P}(H)$ is a s.m. called the image by $f$ of the spectral measure $\mathcal{E}$.

In the all following text, except explicit mention of the opposite, all the considered s.m.'s are s.m.'s on $\mathcal{B}$ for $H$.

When $\mathcal{E}$ is a s.m., the application $X \in H \longmapsto \int e^{i} \cdot d Z_{\mathcal{E}}^{X} \in H$ is a unit operator called unit operator deduced from the s.m. $\mathcal{E}$. In this way, with each s.m. corresponds a unit operator.

Conversely, with a unit operator $U$, we can associate a s.m. in the following way: let $X$ be an element of $H$, then the series $\left(U^{n} X\right)_{n \in \mathbb{Z}}$ is stationary. If we denote by $Z^{X}$ the r.m. associated with this series, we can prove that

i) for all $A$ of $\mathcal{B}$ the application $\mathcal{E}(A): X \in H \longmapsto Z^{X}(A) \in H$ is an orthogonal projector,

ii) the application $\mathcal{E}: A \in \mathcal{B} \longmapsto \mathcal{E}(A) \in \mathcal{P}(H)$ is a s.m. called s.m. associated with the unit operator $U$.

The unit operator deduced from the s.m. $\mathcal{E}$ is $U$.

The s.m. associated with the unit operator $I_{H}$ is the s.m. $\mathcal{E}_{\Pi}$ defined by $A \in \mathcal{B} \longmapsto \delta_{0}(A) I_{H} \in \mathcal{P}(H)$.

If $\mathcal{E}$ is the s.m. associated with the unit operator $U, w(\mathcal{E})$ is the s.m. associated with the unit operator $U^{*}=U^{-1}$.

Two unit operators $U_{1}$ and $U_{2}$ commute if and only if their respectively associated s.m.'s, $\mathcal{E}_{1}$ and $\mathcal{E}_{2}$, commute, that is are such that $\left(\mathcal{E}_{1} A_{1}\right) \circ\left(\mathcal{E}_{2} A_{2}\right)=\left(\mathcal{E}_{2} A_{2}\right) \circ\left(\mathcal{E}_{1} A_{1}\right)$ for all couple $\left(A_{1}, A_{2}\right)$ of elements of $\mathcal{B}$.

If two s.m.'s, $\mathcal{E}_{1}$ and $\mathcal{E}_{2}$, commute, we can affirm that it exists one and only one s.m. on $\mathcal{B} \otimes \mathcal{B}$ for $H$, denoted by $\mathcal{E}_{1} \otimes \mathcal{E}_{2}$, such that $\mathcal{E}_{1} \otimes \mathcal{E}_{2}\left(A_{1} \times A_{2}\right)=\left(\mathcal{E}_{1} A_{1}\right) \circ\left(\mathcal{E}_{2} A_{2}\right)$ for all couple $\left(A_{1}, A_{2}\right)$ of elements of $\mathcal{B}$.

When two s.m.'s $\mathcal{E}_{1}$ and $\mathcal{E}_{2}$ commute we call convolution product of $\mathcal{E}_{1}$ and $\mathcal{E}_{2}$, that we denote $\mathcal{E}_{1} * \mathcal{E}_{2}$, the image by $S$ of $\mathcal{E}_{1} \otimes \mathcal{E}_{2}$. Then we have: $\mathcal{E}_{1} * \mathcal{E}_{2}=\mathcal{E}_{2} * \mathcal{E}_{1}$.

If two unit operators $U_{1}$ and $U_{2}$, of respectively associated s.m.'s $\mathcal{E}_{1}$ and $\mathcal{E}_{2}$, commute, then $\mathcal{E}_{1} * \mathcal{E}_{2}$ is the s.m. associated with the unit operators $U_{1} \circ U_{2}$.

Let end this section by the examen of some algebraic properties.

The s.m. $\mathcal{E}_{\Pi}$ commutes with each s.m. $\mathcal{E}$ and: $\mathcal{E}_{\Pi} * \mathcal{E}=\mathcal{E}$.

If $\mathcal{E}_{1}, \mathcal{E}_{2}$ and $\mathcal{E}_{3}$ are three s.m.'s, on $\mathcal{B}$ for $H$, that commute two by two, then $\mathcal{E}_{1} *\left(\mathcal{E}_{2} * \mathcal{E}_{3}\right)=\left(\mathcal{E}_{1} * \mathcal{E}_{2}\right) * \mathcal{E}_{3}$.

If $f$ and $g$ are two measurable applications from $\Pi$ into itself, then:

i) the application $f \oplus g: \lambda \in \Pi \longmapsto f(\lambda) \oplus g(\lambda) \in \Pi$ is measurable,

ii) when $\mathcal{E}$ is a s.m., the s.m.'s $f(\mathcal{E})$ and $g(\mathcal{E})$ commute and: $(f \oplus g) \mathcal{E}=f(\mathcal{E}) * g(\mathcal{E})$.

\subsection{Order relation in the set of orthogonal projectors}

When $P_{1}$ and $P_{2}$ are two elements of $\mathcal{P}(H)$, the two following assertions are equivalent:

i) $P_{1} \circ P_{2}=P_{1}$,

ii) $i m P_{1} \subset i m P_{2}$.

Then, we say that $P_{1}$ is lower or equal to $P_{2}$, what we will note $P_{1} \ll P_{2}$. The relation $\ll$ is a partial order relation in $\mathcal{P}(H)$. The inclusion in $\mathcal{B}$ is a partial order relation which is transposed in $\mathcal{P}(H)$ by a s.m. as follows:

Lemma 1.5 If $\mathcal{E}$ is a s.m. on $\mathcal{X}$ for $H$, then for all couple $(A, B)$ of elements of $\mathcal{X}$ such that $A \subset B$, we have: $(\mathcal{E} A) \ll(\mathcal{E} B)$. 


\section{The $\alpha$-equivalence of spectral measures}

In this paragraph we define and study a relation which is intended to measure the proximity between spectral measures: the $\alpha$-equivalence.

Definition 2.1 Let $\alpha$ be an element of [0, $\pi$ [. We will say that two s.m.'s $\mathcal{E}_{1}$ and $\mathcal{E}_{2}$ are $\alpha$-equivalent, what we will denote $\mathcal{E}_{1} \stackrel{\alpha}{\sim} \mathcal{E}_{2}$, when for all $B$ of $\mathcal{K}$, we have:

i) $\mathcal{E}_{1}(B) \ll \mathcal{E}_{2}(B \oplus[-\alpha, \alpha])$,

ii) $\mathcal{E}_{2}(B) \ll \mathcal{E}_{1}(B \oplus[-\alpha, \alpha])$.

Remarks.

i) this relation is reflexive and symmetric;

ii) two s.m.'s $\mathcal{E}_{1}$ and $\mathcal{E}_{2}$ 0-equivalent are equal. Indeed, for all $B$ of $\mathcal{K}$, we have $\mathcal{E}_{1}(B)=\mathcal{E}_{2}(B)$. Consequently, for all $X$ of $H$, the r.m.'s $Z_{\mathcal{E}_{1}}^{X}$ and $Z_{\mathcal{E}_{2}}^{X}$ match on $\mathcal{K}$ and so are equal, hence: $\mathcal{E}_{1}=\mathcal{E}_{2}$.

The $\alpha$-equivalence has a property close to transitivity.

Lemma 2.1 When $\left(\alpha_{1}, \alpha_{2}\right)$ is a couple of elements of $\left[0, \pi\left[\right.\right.$ such that $\alpha_{1}+\alpha_{2}<\pi$, if three s.m.'s $\mathcal{E}_{1}$, $\mathcal{E}_{2}$ and $\mathcal{E}_{3}$ are such that $\mathcal{E}_{1} \stackrel{\alpha_{1}}{\sim} \mathcal{E}_{2}$ and $\mathcal{E}_{2} \stackrel{\alpha_{2}}{\sim} \mathcal{E}_{3}$ then: $\mathcal{E}_{1} \stackrel{\alpha_{1}+\alpha_{2}}{\sim} \mathcal{E}_{3}$.

Proof. This comes from the relation $\left[-\alpha_{1}, \alpha_{1}\right] \oplus\left[-\alpha_{2}, \alpha_{2}\right] \subset\left[-\left(\alpha_{1}+\alpha_{2}\right), \alpha_{1}+\alpha_{2}\right]$, and the fact that $B \oplus\left[-\alpha_{i}, \alpha_{i}\right]$, $i=1,2$, is compact as soon as $B$ is compact.

When a s.m. is $\alpha$-equivalent to $\mathcal{E}_{\Pi}$, it is close to $\mathcal{E}_{\Pi}$ in the following sense:

Proposition 2.1 A s.m. $\mathcal{E}$ is $\alpha$-equivalent to $\mathcal{E}_{\Pi}$ if and only if $\mathcal{E}([-\alpha, \alpha])=I_{H}$.

Proof. Let $\mathcal{E}$ be a s.m. such that $\mathcal{E}([-\alpha, \alpha])=I_{H}$. Consider $B$ an element of $\mathcal{K}$. If $0 \in B \oplus[-\alpha, \alpha]$, then $\mathcal{E}(B) \ll I_{H}=\mathcal{E}_{\Pi}(B \oplus[-\alpha, \alpha])$. If $0 \notin B \oplus[-\alpha, \alpha]$, then $B \cap[-\alpha, \alpha]=\emptyset$ and hence $\mathcal{E}(B) \circ \mathcal{E}_{\Pi}(B \oplus[-\alpha, \alpha])=0=$ $\mathcal{E}(B \cap[-\alpha, \alpha])=\mathcal{E}(B) \circ \mathcal{E}([-\alpha, \alpha])=\mathcal{E}(B)$. In both cases we have $\mathcal{E}(B) \ll \mathcal{E}_{\Pi}(B \oplus[-\alpha, \alpha])$.

In order to prove that $\mathcal{E}_{\Pi}(B) \ll \mathcal{E}(B \oplus[-\alpha, \alpha])$, we also consider two cases: $\mathcal{E}_{\Pi}(B)=I_{H}$ and $\mathcal{E}_{\Pi}(B)=0$. In the first case $0 \in B$ and so $[-\alpha, \alpha] \subset B \oplus[-\alpha, \alpha]$, what allows to write $\mathcal{E}_{\Pi}(B)=I_{H}=\mathcal{E}([-\alpha, \alpha]) \ll \mathcal{E}(B \oplus[-\alpha, \alpha])$. In the second case it comes: $\mathcal{E}_{\Pi}(B)=0 \ll \mathcal{E}(B \oplus[-\alpha, \alpha])$.

We then conclude that $\mathcal{E}$ is $\alpha$-equivalent to $\mathcal{E}_{\Pi}$.

The reverse comes from the relations $I_{H}=\mathcal{E}_{\Pi}(\{0\}) \ll \mathcal{E}(\{0\} \oplus[-\alpha, \alpha])=\mathcal{E}([-\alpha, \alpha]) \ll \mathcal{E}(\Pi)=I_{H}$.

The $\alpha$-equivalence has the following property of continuity.

Proposition 2.2 Let $\left(\alpha_{n}\right)_{n \in \mathbb{N}}$ be a sequence of elements of $[0, \pi[$ that decreasingly converges towards $\alpha$, then two s.m.'s $\mathcal{E}_{1}$ and $\mathcal{E}_{2}$ such that $\mathcal{E}_{1} \stackrel{\alpha_{n}}{\sim} \mathcal{E}_{2}$, for all integer $n$, are $\alpha$-equivalent.

Proof. Let $B$ be a compact set of $\Pi$. As $\left(B \oplus\left[-\alpha_{n}, \alpha_{n}\right]\right)_{n \in \mathbb{N}}$ is a decreasing sequence of elements of $\mathcal{B}$, the lemma 1.4 allows to write

$$
\lim _{n} \mathcal{E}_{2}\left(B \oplus\left[-\alpha_{n}, \alpha_{n}\right]\right) X=\mathcal{E}_{2}\left(\cap_{n \in \mathbb{N}}\left(B \oplus\left[-\alpha_{n}, \alpha_{n}\right]\right)\right) X=\mathcal{E}_{2}(B \oplus[-\alpha, \alpha]) X,
$$

hence

$$
\lim _{n} \mathcal{E}_{1}(B)\left(\mathcal{E}_{2}\left(B \oplus\left[-\alpha_{n}, \alpha_{n}\right]\right) X\right)=\mathcal{E}_{1}(B)\left(\mathcal{E}_{2}(B \oplus[-\alpha, \alpha]) X\right) .
$$

Besides, as $\mathcal{E}_{1}(B) \circ\left(\mathcal{E}_{2}\left(B \oplus\left[-\alpha_{n}, \alpha_{n}\right]\right)\right)=\mathcal{E}_{1}(B)$, the above equality becomes $\lim _{n} \mathcal{E}_{1}(B) X=\mathcal{E}_{1}(B)\left(\left(\mathcal{E}_{2}(B \oplus[-\alpha, \alpha]) X\right)\right)$ hence $\mathcal{E}_{1}(B) \ll\left(\mathcal{E}_{2}(B \oplus[-\alpha, \alpha])\right.$. The relation $\mathcal{E}_{2}(B) \ll \mathcal{E}_{1}(B \oplus[-\alpha, \alpha])$ can be proved in a same way, changing the roles of $\mathcal{E}_{1}$ and of $\mathcal{E}_{2}$. This completes the proof.

Let now examine how the convolution product keeps the property of $\alpha$-equivalence. Consider first the case of a s.m. concentrated on a finite number of elements of $\Pi .^{2}$

Lemma 2.2 Let two s.m.'s $\mathcal{E}_{1}$ and $\mathcal{E}_{2}, \alpha$-equivalent, and $\mathcal{E}$ a third s.m. concentrated on a finite number of elements of $\Pi$ that commute with $\mathcal{E}_{1}$ and with $\mathcal{E}_{2}$, then the s.m.'s $\mathcal{E} * \mathcal{E}_{1}$ and $\mathcal{E} * \mathcal{E}_{2}$ are $\alpha$-equivalent.

Proof. There exists a finite family $\left\{\lambda_{j} ; j \in J\right\}$ of elements of $\Pi$ such that $\mathcal{E}\left(\left\{\lambda_{j} ; j \in J\right\}\right)=I_{H}$.

Let $B$ be a compact subset of $\Pi$, we have:

${ }^{2}$ Let recall that a s.m. $\mathcal{E}$ is said to be concentrated on a finite number of elements of $\Pi$ when there exists a finite family $\left\{\lambda_{j} ; j \in J\right\}$ of elements of $\Pi$ such that $\mathcal{E}\left(\left\{\lambda_{j} ; j \in J\right\}\right)=I_{H}$, if $\mathcal{E}^{\prime}$ is a second s.m. which commutes with $\mathcal{E}$, then, for all $A$ of $\mathcal{B}$, we have: $\mathcal{E} * \mathcal{E}^{\prime}(A)=\sum_{j \in J} \mathcal{E}\left(\left\{\lambda_{j}\right\}\right) \circ \mathcal{E}^{\prime}\left(A \ominus \lambda_{j}\right)$. 


$$
\left(\mathcal{E} * \mathcal{E}_{1}(B)\right) \circ\left(\mathcal{E} * \mathcal{E}_{2}(B \oplus[-\alpha, \alpha])\right)=\sum_{j \in J} \sum_{j^{\prime} \in J} \mathcal{E}\left(\left\{\lambda_{j}\right\}\right) \circ \mathcal{E}_{1}\left(B \ominus \lambda_{j}\right) \circ \mathcal{E}\left(\left\{\lambda_{j^{\prime}}\right\}\right) \circ \mathcal{E}_{2}\left((B \oplus[-\alpha, \alpha]) \ominus \lambda_{j^{\prime}}\right) .
$$

Moreover, as $\mathcal{E}_{1}\left(B \ominus \lambda_{j}\right) \circ \mathcal{E}_{2}\left(\left(B \ominus \lambda_{j}\right) \oplus[-\alpha, \alpha]\right)=\mathcal{E}_{1}\left(B \ominus \lambda_{j}\right)$ we can write:

$$
\left(\mathcal{E} * \mathcal{E}_{1}(B)\right) \circ\left(\mathcal{E} * \mathcal{E}_{2}(B \oplus[-\alpha, \alpha])\right)=\sum_{j \in J} \mathcal{E}\left(\left\{\lambda_{j}\right\}\right) \circ \mathcal{E}_{1}\left(B \ominus \lambda_{j}\right)=\mathcal{E} * \mathcal{E}_{1}(B) .
$$

In a similar way, we can prove that $\left(\mathcal{E} * \mathcal{E}_{2}(B)\right) \circ\left(\mathcal{E} * \mathcal{E}_{1}(B \oplus[-\alpha, \alpha])\right)=\mathcal{E} * \mathcal{E}_{2}(B)$.

The following result also deals with the convolution product.

Lemma 2.3 If $\mathcal{E}$ is a s.m. $\alpha$-equivalent to $\mathcal{E}_{\Pi}$ and $\mathcal{E}^{\prime}$ a second s.m. which commutes with $\mathcal{E}$, then for all $B$ of $\mathcal{B}$ we have:

i) $\mathcal{E}^{\prime} * \mathcal{E}(B) \ll \mathcal{E}^{\prime}(B \oplus[-\alpha, \alpha])$,

ii) $\mathcal{E}^{\prime}(B) \ll \mathcal{E}^{\prime} * \mathcal{E}(B \oplus[-\alpha, \alpha])$.

Proof. Let consider $B$ an element of $\mathcal{B}$. From $\left((\Pi \times[-\alpha, \alpha]) \cap\left(S^{-1} B\right)\right) \subset((B \oplus[-\alpha, \alpha]) \times[-\alpha, \alpha])$ we deduce:

$$
\mathcal{E}^{\prime} * \mathcal{E}(B)=\mathcal{E}^{\prime} \otimes \mathcal{E}\left((\Pi \times[-\alpha, \alpha]) \cap\left(S^{-1} B\right)\right) \ll \mathcal{E}^{\prime} \otimes \mathcal{E}((B \oplus[-\alpha, \alpha]) \times[-\alpha, \alpha])=\mathcal{E}^{\prime}(B \oplus[-\alpha, \alpha]) .
$$

Moreover, as $(B \times[-\alpha, \alpha]) \subset S^{-1}(B \oplus[-\alpha, \alpha])$, we can write:

$$
\mathcal{E}^{\prime}(B)=\mathcal{E}^{\prime} \otimes \mathcal{E}(B \times[-\alpha, \alpha]) \ll \mathcal{E}^{\prime} \otimes \mathcal{E}\left(S^{-1}(B \oplus[-\alpha, \alpha])\right)=\mathcal{E}^{\prime} * \mathcal{E}(B \oplus[-\alpha, \alpha]),
$$

and the proof is complete.

As $\mathcal{K}$ is a subset of $\mathcal{B}$, from this last result we deduce the

Corollary 2.1 If a s.m. $\mathcal{E}$ is $\alpha$-equivalent to $\mathcal{E}_{\Pi}$, then for all s.m. $\mathcal{E}^{\prime}$ that commutes with $\mathcal{E}$, we have: $\mathcal{E} * \mathcal{E}^{\prime} \stackrel{\alpha}{\sim} \mathcal{E}^{\prime}$.

Now we will examine how a s.m. can be approximated by a s.m. concentrated on a finite number of elements of $\Pi$ thanks to a partition of $\Pi$.

For this, consider the measurable application $\mathcal{L}_{k}=\sum_{q=0}^{q=k-1}\left(-\pi+q \frac{2 \pi}{k}\right) 1_{\left[-\pi+q \frac{2 \pi}{k},-\pi+(q+1) \frac{2 \pi}{k}\right.}, \mathrm{k}$ being an integer $\geq 3$. Hence we have the equality $\left(\mathcal{L}_{k} \oplus w\right)^{-1}\left(\left[-\frac{2 \pi}{k}, \frac{2 \pi}{k}\right]\right)=\Pi$, that plays a fundamental role for the proof of the

Lemma 2.4 When $k$ is an integer $\geq 3$, for all s.m. $\mathcal{E}$ we can say that:

i) $\left(\mathcal{L}_{k} \mathcal{E}\right) *(w \mathcal{E})$ is $\frac{2 \pi}{k}$-equivalent to $\mathcal{E}_{\Pi}$;

ii) $\mathcal{L}_{k} \mathcal{E}$ is $\frac{2 \pi}{k}$-equivalent to $\mathcal{E}$.

Proof. The point i) is a consequence of the equality $\left(\mathcal{L}_{k} \oplus w\right)^{-1}\left(\left[-\frac{2 \pi}{k}, \frac{2 \pi}{k}\right]\right)=\Pi$ and of proposition 2.1.

The point ii) is deduced from the point i) thanks to corollary 2.1 .

It is possible to generalize lemma 2.2 and corollary 2.1.

Proposition 2.3 Let two s.m.'s $\mathcal{E}_{1}$ and $\mathcal{E}_{2}, \alpha$-equivalent, and $\mathcal{E}$ a third s.m. which commutes with $\mathcal{E}_{1}$ and with $\mathcal{E}_{2}$, then the s.m.'s $\mathcal{E} * \mathcal{E}_{1}$ and $\mathcal{E} * \mathcal{E}_{2}$ are $\alpha$-equivalent.

Proof. Let two s.m.'s $\mathcal{E}_{1}$ and $\mathcal{E}_{2}$ be $\alpha$-equivalent and $\mathcal{E}$ a third s.m. which commutes with $\mathcal{E}_{1}$ and with $\mathcal{E}_{2}$. Consider an integer $k \geq\left[\frac{4 \pi}{\pi-\alpha}\right]+1$.

The successive use of lemma 2.4 and of corollary 2.1 let us write $\left(\mathcal{L}_{k} \mathcal{E}\right) * \mathcal{E}_{1} \stackrel{\frac{2 \pi}{k}}{\sim} \mathcal{E} * \mathcal{E}_{1}$ and $\left(\mathcal{L}_{k} \mathcal{E}\right) * \mathcal{E}_{2} \stackrel{\frac{2 \pi}{k}}{\sim} \mathcal{E} * \mathcal{E}_{2}$.

From the fact that $\mathcal{L}_{k} \mathcal{E}$ is a s.m. concentrated on a finite number of elements of $\Pi$, the lemma 2.2 allows to write $\left(\mathcal{L}_{k} \mathcal{E}\right) * \mathcal{E}_{1} \stackrel{\alpha}{\sim}\left(\mathcal{L}_{k} \mathcal{E}\right) * \mathcal{E}_{2}$.

From the above relations and from lemma 2.1, we can say that the s.m.'s $\mathcal{E} * \mathcal{E}_{1}$ and $\mathcal{E} * \mathcal{E}_{2}$ are $\left(\alpha+\frac{4 \pi}{k}\right)-$ equivalent, for all integer $k \geq\left[\frac{4 \pi}{\pi-\alpha}\right]+1$. From proposition 2.2, we finally deduce the $\alpha$-equivalence of the s.m.'s $\mathcal{E} * \mathcal{E}_{1}$ and $\mathcal{E} * \mathcal{E}_{2}$.

The previous result and corollary 2.1 allow to establish a link between the $\alpha$-equivalence of two s.m.'s $\mathcal{E}_{1}$ and $\mathcal{E}_{2}$, that commute, and the $\alpha$-equivalence of the s.m.'s $\left(w \mathcal{E}_{1}\right) * \mathcal{E}_{2}$ and $\mathcal{E}_{\Pi}$.

Proposition 2.4 Two s.m.'s $\mathcal{E}_{1}$ and $\mathcal{E}_{2}$ that commute are $\alpha$-equivalent if and only if the s.m.'s $\left(w \mathcal{E}_{1}\right) * \mathcal{E}_{2}$ and $\mathcal{E}_{\Pi}$ are $\alpha$-equivalent.

We can now give a new formulation of the $\alpha$-equivalence when the s.m.'s commute:

Proposition 2.5 Two s.m.'s $\mathcal{E}_{1}$ and $\mathcal{E}_{2}$ which commute are $\alpha$-equivalent if and only if for all $B$ of $\mathcal{B}$, we have:

i) $\mathcal{E}_{1}(B) \ll \mathcal{E}_{2}(B \oplus[-\alpha, \alpha])$,

ii) $\mathcal{E}_{2}(B) \ll \mathcal{E}_{1}(B \oplus[-\alpha, \alpha])$.

Proof. If relations i) and ii) are verified for all $B$ of $\mathcal{B}$, the $\alpha$-equivalence comes from the fact that $\mathcal{K} \subset \mathcal{B}$. 
Conversely, consider two s.m.'s $\mathcal{E}_{1}$ and $\mathcal{E}_{2}$ which commute and are $\alpha$-equivalent. From proposition 2.4, we have $\left(w \mathcal{E}_{1}\right) * \mathcal{E}_{2} \stackrel{\alpha}{\sim} \mathcal{E}_{\Pi}$. Since the s.m.'s $\mathcal{E}_{1}$ and $\left(w \mathcal{E}_{1}\right) * \mathcal{E}_{2}$ commute, the lemma 2.3 let us write, for all $B$ of $\mathcal{B}: \mathcal{E}_{2}(B) \ll$ $\mathcal{E}_{1}(B \oplus[-\alpha, \alpha])$ and $\mathcal{E}_{1}(B) \ll \mathcal{E}_{2}(B \oplus[-\alpha, \alpha])$, and the proof is complete.

Remark. When two s.m.'s $\mathcal{E}_{1}$ and $\mathcal{E}_{2}$ commute and are $\alpha$-equivalent, if $\alpha<\frac{\pi}{2}$, for all Borel set $B$, we have

$$
\mathcal{E}_{1}(B) \ll \mathcal{E}_{2}(B \oplus[-\alpha, \alpha]) \ll \mathcal{E}_{1}(B \oplus[-2 \alpha, 2 \alpha]) .
$$

As soon as $\alpha$ is small, the Borel sets $B, B \oplus[-\alpha, \alpha]$ and $B \oplus[-2 \alpha, 2 \alpha]$ can be qualified of close together. We can say the same for the projectors $\mathcal{E}_{1}(B)$ and $\mathcal{E}_{1}(B \oplus[-2 \alpha, 2 \alpha])$, and for the projectors $\mathcal{E}_{2}(B)$ and $\mathcal{E}_{2}(B \oplus[-\alpha, \alpha])$. From the above relations $\mathcal{E}_{2}(B \oplus[-\alpha, \alpha])$ is between $\mathcal{E}_{1}(B)$ and $\mathcal{E}_{1}(B \oplus[-2 \alpha, 2 \alpha])$, so it is close to $\mathcal{E}_{1}(B)$. We then can deduce the proximity between $\mathcal{E}_{1}(B)$ and $\mathcal{E}_{2}(B)$.

\section{Unit operators and $\alpha$-equivalence}

The s.m. associated with the unit operator $I_{H}$ is $\mathcal{E}_{\Pi}$, that is the s.m. concentrated on 0 . It is natural to think that the s.m. associated with a unit operator close to $I_{H}$ has an analogous property. We will first examine such a result.

For this, it is necessary to use a preliminary result about the application $C: \lambda \in \Pi \longmapsto \cos (\lambda) \in \mathbb{R}$, which is proved using elementary tools of measure theory:

Lemma 3.1 If $\mu$ is a probability measure defined on $\mathcal{B}$ such that $1-\varepsilon^{2} \leq \int C d \mu$, where $\varepsilon$ is an element of $] 0,1[$, then: $1-\varepsilon \leq \mu([-2 \sqrt{\varepsilon}, 2 \sqrt{\varepsilon}])$.

Consequently,

Proposition 3.1 If $U$ is a unit operator of $H$, of associated s.m. $\mathcal{E}$, such that $\left\|U-I_{H}\right\|_{\mathcal{L}} \leq \varepsilon$, where $\varepsilon$ is an element of $] 0,1\left[\right.$, then: $\mathcal{E}\left(\left[-2^{\frac{3}{4}} \sqrt{\varepsilon}, 2^{\frac{3}{4}} \sqrt{\varepsilon}\right]\right)=I_{H}$.

Proof. If $X$ is a normed element of $H$, we can write: $\|U(X)-X\|^{2} \leq\left\|U-I_{H}\right\|_{\mathcal{L}}^{2} \leq \varepsilon^{2}$.

Developping this last point, we obtain

$$
1-\left(\frac{\varepsilon}{\sqrt{2}}\right)^{2} \leq \int C d \mu_{Z_{\varepsilon}^{X}}
$$

As $\mu_{Z_{\mathcal{E}}^{X}}$ is a "probability measure" the use of lemma 3.1 allows us to write:

$$
\left\|\left(\mathcal{E} \complement\left[-2^{\frac{3}{4}} \sqrt{\varepsilon}, 2^{\frac{3}{4}} \sqrt{\varepsilon}\right]\right) X\right\|^{2}=\left\|Z_{\mathcal{E}}^{X}\left(\complement\left[-2^{\frac{3}{4}} \sqrt{\varepsilon}, 2^{\frac{3}{4}} \sqrt{\varepsilon}\right]\right)\right\|^{2}=\mu_{Z_{\mathcal{E}}^{X}}\left(\complement\left[-2^{\frac{3}{4}} \sqrt{\varepsilon}, 2^{\frac{3}{4}} \sqrt{\varepsilon}\right]\right) \leq \frac{\varepsilon}{\sqrt{2}} .
$$

Since the norm of an orthogonal projector is either 1 or 0 , we have $\mathcal{E}\left(\left[-2^{\frac{3}{4}} \sqrt{\varepsilon}, 2^{\frac{3}{4}} \sqrt{\varepsilon}\right]\right)=I_{H}$.

We have just established that the s.m. associated with a unit operator close to the identity is concentrated around 0 . The result that we now will examine constitutes, in some way, the reverse: when a s.m. is concentrated around 0 , the deduced unit operator is close to identity.

Proposition 3.2 If the s.m. $\mathcal{E}$, associated with a unit operator $U$, is such that $\mathcal{E}[-\varepsilon, \varepsilon]=I_{H}$, where $\varepsilon$ is an element of $\left[0, \pi\left[\right.\right.$, then $\left\|U-I_{H}\right\|_{\mathcal{L}} \leq \sqrt{2} \sqrt{1-\cos (\varepsilon)} \leq \varepsilon$.

Proof. Let $X$ be a normed element of $H$, if we remark that $\mu_{Z_{\mathcal{E}}^{X}}(\mathcal{C}[-\varepsilon, \varepsilon])=0$, we can write:

$$
\|U(X)-X\|^{2}=2-2 \int C d \mu_{Z_{\mathcal{E}}^{X}}=2-2 \int 1_{[-\varepsilon, \varepsilon]} C d \mu_{Z_{\mathcal{E}}^{X}} \leq 2(1-\cos (\varepsilon)) \leq \varepsilon^{2},
$$

so the property stands.

The proximity between unit operators implies that of associated s.m.'s, it is the object of the

Theorem 3.1 If two unit operators $U_{1}$ and $U_{2}$ commute and are such that $\left\|U_{2}-U_{1}\right\|_{\mathcal{L}} \leq \varepsilon$, $\varepsilon$ being an element of ]0,1[, then the respectively associated s.m.'s $\mathcal{E}_{1}$ and $\mathcal{E}_{2}$ are $2^{\frac{3}{4}} \sqrt{\varepsilon}$-equivalent.

Proof. To prove it, we can use the relations $\left\|U_{1}^{-1} \circ U_{2}-I_{H}\right\|_{\mathcal{L}}=\left\|U_{2}-U_{1}\right\|_{\mathcal{L}} \leq \varepsilon$ and then propositions 3.1, 2.1 and 2.4 .

Remark. The hypothesis of commutativity is necessary, more precisely, the s.m.'s $\mathcal{E}_{1}$ and $\mathcal{E}_{2}$, associated with two unit operators $U_{1}$ and $U_{2}$ such that $\left\|U_{2}-U_{1}\right\|_{\mathcal{L}} \leq \varepsilon$, are not necessarily $2^{\frac{3}{4}} \sqrt{\varepsilon}$-equivalent when there is not commutativity. Examples can be given.

We have just seen that, when there is commutativity, the proximity between unit operators induces that of the associated s.m.'s. Conversely, if two s.m.'s are close together, the same happens for the induced unit operators, indeed:

Theorem 3.2 If two s.m.'s $\mathcal{E}_{1}$ and $\mathcal{E}_{2}$ that commute are $\alpha$-equivalent, then we have the inequality: $\left\|U_{1}-U_{2}\right\|_{\mathcal{L}} \leq \alpha$, $U_{1}$ and $U_{2}$ denoting the unit operators respectively deduced from $\mathcal{E}_{1}$ and from $\mathcal{E}_{2}$.

Proof. The result is a consequence of propositions 2.4, 2.1, 3.2 and of the fact that $\left\|U_{1}^{-1} \circ U_{2}-I_{H}\right\|_{\mathcal{L}}=\left\|U_{2}-U_{1}\right\|_{\mathcal{L}}$ 


\section{Applications to stationary processes}

The following application allows to establish a duality between the quasi-periodicity of a process and the concentration of the spectral measures.

Let us begin by specifying some spectral elements that we associate with a stationary c.r.f. $\left(X_{g}\right)_{g \in G}$. We can show (cf. Boudou and Romain, 2002) that, if $Z$ denotes its associated r.m., it exists one and only one s.m. $\mathcal{E}$, on $\mathcal{B}_{\widehat{G}}$ for $H_{Z}$, called s.m. associated with $Z$, such that $\mathcal{E}(A)\left(\int \varphi d Z\right)=\int 1_{A} \varphi d Z$ for all $(A, \varphi)$ of $\mathcal{B}_{\widehat{G}} \times L^{2}\left(\widehat{G}, \mathcal{B}_{\widehat{G}}, \mu_{Z}\right)$.

In Boudou (2007), a family of unit operators is associated with a s.m.. In the case of the s.m. previously defined, this can be summarised in the following way:

- for all $(\gamma, g)$ of $\widehat{G} \times G$, we denote $\bar{g}(\gamma)$ the unique element of $\Pi$ such that $e^{i \bar{g}(\gamma)}=(\gamma, g)_{\widehat{G} G}$; the application $\bar{g}: \gamma \in \widehat{G} \longmapsto \bar{g}(\gamma) \in \Pi$ is measurable;

- for all $g$ of $G$ we note $U_{g}$ the unit operator of $H_{Z}$ deduced from the s.m. $\bar{g}(\mathcal{E})$.

We will call $\left\{U_{g} ; g \in G\right\}$ the family of the unit operators of $H_{Z}$ deduced from the stationary c.r.f. $\left(X_{g}\right)_{g \in G}$.

We can then assert (Boudou, 2007) that

i) for all $\left(g, g^{\prime}\right)$ of $G \times G$, we have $U_{g} \circ U_{g^{\prime}}=U_{g+g^{\prime}}$;

ii) for all $X$ of $H_{Z},\left(U_{g}(X)\right)_{g \in G}$ is a stationary c.r.f. of associated r.m. $Z_{\mathcal{E}}^{X}$.

So, when $\varphi$ is an element of $L^{2}\left(\widehat{G}, \mathcal{B}_{\widehat{G}}, \mu_{Z}\right),\left(U_{g}\left(\int \varphi d Z\right)\right)_{g \in G}$ is a stationary c.r.f. image of $\left(X_{g}\right)_{g \in G}$ by the filter $\varphi$.

From these recalls, we can now introduce our following new concept:

Definition 4.1 A stationary c.r.f. $\left(X_{g}\right)_{g \in G}$, for which $\left\{U_{g} ; g \in G\right\}$ is the family of the deduced unit operators, has the property of $\left(\alpha, g_{1}\right)$-proximity, $\left(\alpha, g_{1}\right)$ being an element of $] 0,1\left[\times G\right.$, when $\left\|U_{g_{1}}-I_{H_{Z}}\right\|_{\mathcal{L}} \leq \alpha$.

Remark. This means that for all stationary c.r.f. of type $\left(\int(., g)_{\widehat{G} G} \varphi(.) d Z\right)_{g \in G}$, where $\varphi$ is an element of $L^{2}\left(\mu_{Z}\right)$, we have, for all $g$ of $G$ :

$$
\left\|\int\left(., g+g_{1}\right)_{\widehat{G} G} \varphi(.) d Z-\int(., g)_{\widehat{G} G} \varphi(.) d Z\right\| \leq \alpha\left\|\int \varphi d Z\right\| .
$$

Conversely, if for all element $\varphi$ of $L^{2}\left(\mu_{Z}\right)$ and for all $g$ of $G$, this inequality stands, then the stationary c.r.f. $\left(X_{g}\right)_{g \in G}$ has the property of $\left(\alpha, g_{1}\right)$-proximity.

In the frequency domain, the $\left(\alpha, g_{1}\right)$-proximity of a stationary c.r.f. takes the form of a concentration of the associated r.m.:

Proposition 4.1 If a stationary c.r.f. $\left(X_{g}\right)_{g \in G}$, of associated r.m. $Z$ has the property of $\left(\alpha, g_{1}\right)-$ proximity, $\left(\alpha, g_{1}\right)$ being an element of $] 0,1\left[\times G\right.$, then: $Z\left(\complement\left(\bar{g}_{1}^{-1}\left(\left[-\alpha^{\prime}, \alpha^{\prime}\right]\right)\right)\right)=0$, where $\alpha^{\prime}=2^{\frac{3}{4}} \sqrt{\alpha}$.

Proof. Let denote $\mathcal{E}$ the s.m. associated with $Z$ and $\left\{U_{g} ; g \in G\right\}$ the family of the unit operators deduced from the stationary c.r.f. $\left(X_{g}\right)_{g \in G}$. From the definition of the property of $\left(\alpha, g_{1}\right)$-proximity, we have $\left\|U_{g_{1}}-I_{H_{Z}}\right\|_{\mathcal{L}} \leq \alpha$. Hence, according to the proposition 3.1: $\mathcal{E}\left(\bar{g}_{1}^{-1}\left(\left[-\alpha^{\prime}, \alpha^{\prime}\right]\right)\right)=I_{H_{Z}}$, where $\alpha^{\prime}=2^{\frac{3}{4}} \sqrt{\alpha}$. In other words, we have: $\mathcal{E}\left(\complement\left(\bar{g}_{1}^{-1}\left(\left[-\alpha^{\prime}, \alpha^{\prime}\right]\right)\right)\right)=0$. Then the definition of the s.m. associated with a r.m. allows us to write:

$Z\left(\complement\left(\bar{g}_{1}^{-1}\left(\left[-\alpha^{\prime}, \alpha^{\prime}\right]\right)\right)\right)=\int 1_{\complement}\left({\overline{g_{1}}}^{-1}\left(\left[-\alpha^{\prime}, \alpha^{\prime}\right]\right)\right) 1_{\widehat{G}} d Z=\mathcal{E}\left(\complement\left({\overline{g_{1}}}^{-1}\left(\left[-\alpha^{\prime}, \alpha^{\prime}\right]\right)\right)\right)\left(\int 1_{\widehat{G}} d Z\right)=0$, hence the property.

Conversely, the concentration of the r.m. implies a property of $\left(\alpha, g_{1}\right)$-proximity:

Proposition 4.2 If the r.m. Z, associated with a stationary c.r.f. $\left(X_{g}\right)_{g \in G}$, is such that $Z\left(\complement\left(\bar{g}_{1}-1([-\alpha, \alpha])\right)\right)=0$, $\left(\alpha, g_{1}\right)$ being an element of $] 0,1\left[\times G\right.$, then $\left(X_{g}\right)_{g \in G}$ has the property of $\left(\alpha, g_{1}\right)$-proximity.

Proof. Let denote by $\mathcal{E}$ the s.m., on $\mathcal{B}_{\widehat{G}}$ for $H_{Z}$, associated with $Z$, the hypotheses allow us to write, for all $\varphi$ of $L^{2}\left(\mu_{Z}\right)$ :

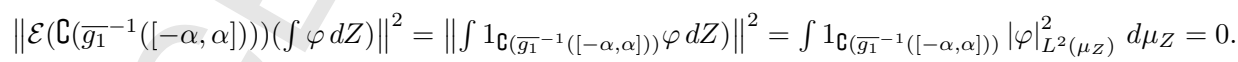

The last equality comes from the fact that $\mu_{Z}\left(\complement\left({\overline{g_{1}}}^{-1}([-\alpha, \alpha])\right)\right)=0$. We can deduce from this that $\mathcal{E}\left(\complement\left(\bar{g}_{1}-1([-\alpha, \alpha])\right)\right)=$ 0 , then that $\left.\overline{g_{1}} \mathcal{E}([-\alpha, \alpha])\right)=I_{H_{Z}}$. As $U_{g_{1}}$ is the unit operator deduced from the s.m. $\overline{g_{1}} \mathcal{E},\left\{U_{g} ; g \in G\right\}$ denoting the family of unit operators of $H_{Z}$ deduced from the stationary c.r.f. $\left(X_{g}\right)_{g \in G}$, the proposition 3.2 allows us to write that $\left\|U_{g_{1}}-I_{H_{Z}}\right\|_{\mathcal{L}} \leq \alpha$, and then to conclude.

In the case where $G$ is equal to $\mathbb{R}$ and $g_{1}$ to $\Delta$, element of $\mathbb{R}_{+}^{*}$, the proposition 4.1 becomes

Corollary 4.1 If a stationary c.r.f. $\left(X_{t}\right)_{t \in \mathbb{R}}$, of associated r.m. $Z$, has the property of $(\alpha, \Delta)-$ proximity, $(\alpha, \Delta)$ being an element of $] 0,1\left[\times \mathbb{R}_{+}^{*}\right.$, then $Z\left(\complement\left(\cup_{n \in \mathbb{Z}}\left[n \frac{2 \pi}{\Delta}-\frac{\alpha^{\prime}}{\Delta}, n \frac{2 \pi}{\Delta}+\frac{\alpha^{\prime}}{\Delta}\right]\right)=0\right.$, where $\alpha^{\prime}=2^{\frac{3}{4}} \sqrt{\alpha}$.

The proposition 4.2 then becomes 
Corollary 4.2 If the r.m. $Z$, associated with a stationary c.r.f. $\left(X_{t}\right)_{t \in \mathbb{R}}$, is such that $Z\left(\complement\left(\cup_{n \in \mathbb{Z}}\left[n \frac{2 \pi}{\Delta}-\frac{\alpha}{\Delta}, n \frac{2 \pi}{\Delta}+\frac{\alpha}{\Delta}\right]\right)\right)=0,(\alpha, \Delta)$ being an element of $] 0,1\left[\times \mathbb{R}_{+}^{*}\right.$, then for all filtered $\left(Y_{t}\right)_{t \in \mathbb{R}}$, of $\left(X_{t}\right)_{t \in \mathbb{R}}$, we have $\left\|Y_{t+\Delta}-Y_{t}\right\| \leq \alpha\left\|Y_{0}\right\|$, and this for all real $t$.

Finally, when $G$ is equal to $\mathbb{Z}$ and when $g_{1}$ is an element $q$ of $\mathbb{N}^{*}$, the proposition 4.1 expresses then as

Corollary 4.3 If a stationary series $\left(X_{n}\right)_{n \in \mathbb{Z}}$, of associated r.m. $Z$ has the property of $(\alpha, q)$-proximity, $(\alpha, q)$ being an element of $] 0,1\left[\times \mathbb{N}^{*}\right.$, then: $Z\left(\complement\left(\cup_{k=0, \ldots, q-1}\left(\lambda_{k} \oplus\left[-\frac{\alpha^{\prime}}{q}, \frac{\alpha^{\prime}}{q}\right]\right)\right)=0\right.$, where $\alpha^{\prime}=2^{\frac{3}{4}} \sqrt{\alpha}$.

Regarding the proposition 4.2 , it becomes

Corollary 4.4 If the r.m. $Z$, associated with a stationary series $\left(X_{n}\right)_{n \in \mathbb{Z},}$ is such that $Z\left(\complement\left(\cup_{k=0, \ldots, q-1}\left(\lambda_{k} \oplus\left[-\frac{\alpha}{q}, \frac{\alpha}{q}\right]\right)\right)=0,(\alpha, q)\right.$ being an element of $] 0,1\left[\times \mathbb{N}^{*}\right.$, then for all filtered $\left(Y_{n}\right)_{n \in \mathbb{Z}}$ of $\left(X_{n}\right)_{n \in \mathbb{Z}}$, we have $\left\|Y_{n+q}-Y_{n}\right\| \leq \alpha\left\|Y_{0}\right\|$, for all $n$ of $\mathbb{Z}$.

\section{$5 \quad$ Numerical illustration}

Let consider a series $\left(X_{n}^{\prime}\right)_{n \in \mathbb{Z}}$ whose spectrum is $\operatorname{spect}\left(\left(X_{n}^{\prime}\right)_{n \in \mathbb{Z}}\right)=\{-2 \pi / 3,-2 \pi / 3 \pm \alpha, 0, \pm \alpha, 2 \pi / 3,2 \pi / 3 \pm \alpha\}$.

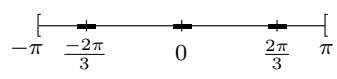

When $\alpha$ is small enough, it is close to a 3 -periodic series $\left(X_{n}\right)_{n \in \mathbb{Z}}$ whose spectrum is $\operatorname{spect}\left(\left(X_{n}\right)_{n \in \mathbb{Z}}\right)=\{-2 \pi / 3,0,2 \pi / 3\}$.

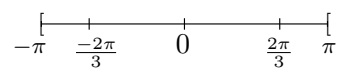

The spectral measure associated with $\left(X_{n}^{\prime}\right)_{n \in \mathbb{Z}}$ can then be writen $\mathcal{E}^{\prime}=\delta_{-\frac{2 \pi}{3}-\alpha}(.) P_{1}^{\prime}+\delta_{-\frac{2 \pi}{3}}(.) P_{2}^{\prime}+\delta_{-\frac{2 \pi}{3}+\alpha}(.) P_{3}^{\prime}+$ $\delta_{-\alpha}(.) P_{4}^{\prime}+\delta_{0}(.) P_{5}^{\prime}+\delta_{\alpha}(.) P_{6}^{\prime}+\delta_{\frac{2 \pi}{3}-\alpha}(.) P_{7}^{\prime}+\delta_{\frac{2 \pi}{3}}(.) P_{8}^{\prime}+\delta_{\frac{2 \pi}{3}+\alpha}(.) P_{9}^{\prime}$.

The associated operator $U^{\prime}=e^{i\left(-\frac{2 \pi}{3}-\alpha\right)} P_{1}^{\prime}+e^{-\frac{2 \pi}{3} i} P_{2}^{\prime}+e^{i\left(-\frac{2 \pi}{3}+\alpha\right)} P_{3}^{\prime}+e^{-i \alpha} P_{4}^{\prime}+P_{5}^{\prime}+e^{i \alpha} P_{6}^{\prime}+e^{i\left(\frac{2 \pi}{3}-\alpha\right)} P_{7}^{\prime}+e^{i \frac{2 \pi}{3}} P_{8}^{\prime}+$ $e^{i\left(\frac{2 \pi}{3}+\alpha\right)} P_{9}^{\prime}$ is such that $U^{\prime 3}$ is close to the identity operator $I$. In a same way, as $X_{n+3}=X_{n}, X_{n+3}^{\prime}$ is close to $X_{n}^{\prime}$.

It is this quasi-periodicity that we will illustrate numerically. The $P_{j}^{\prime}$ 's can be chosen as the orthogonal projectors of a self-adjoint operator generated from random variables. We will choose $Z_{j}=P_{j} 1$ and $Z_{j}^{\prime}=P_{j}^{\prime} 1$, where 1 is the vector of ones. then we can write $X_{n}^{\prime}=e^{i n\left(-\frac{2 \pi}{3}-\alpha\right)} Z_{1}^{\prime}+e^{-i n \frac{2 \pi}{3}} Z_{2}^{\prime}+e^{i n\left(-\frac{2 \pi}{3}+\alpha\right)} Z_{3}^{\prime}+e^{-i n \alpha} Z_{4}^{\prime}+Z_{5}^{\prime}+e^{i n \alpha} Z_{6}^{\prime}+$ $e^{i n\left(\frac{2 \pi}{3}-\alpha\right)} Z_{7}^{\prime}+e^{i n \frac{2 \pi}{3}} Z_{8}^{\prime}+e^{i n\left(\frac{2 \pi}{3}+\alpha\right)} Z_{9}^{\prime}$, and $X_{n+3}^{\prime}=e^{i(n+3)\left(-\frac{2 \pi}{3}-\alpha\right)} Z_{1}^{\prime}+e^{-i(n+3) \frac{2 \pi}{3}} Z_{2}^{\prime}+e^{i(n+3)\left(-\frac{2 \pi}{3}+\alpha\right)} Z_{3}^{\prime}+e^{-i(n+3) \alpha} Z_{4}^{\prime}+$ $Z_{5}^{\prime}+e^{i(n+3) \alpha} Z_{6}^{\prime}+e^{i(n+3)(2 \pi / 3-\alpha)} Z_{7}^{\prime}+e^{2 i(n+3) \pi / 3} Z_{8}^{\prime}+e^{i(n+3)(2 \pi / 3+\alpha)} Z_{9}^{\prime}$.

For $\alpha=0.1$, the values of $X_{1}^{\prime}$ and $X_{4}^{\prime}$ are rather different, when $\alpha=0.01$, they become closer, and when $\alpha=0.001$ and 0.0001 , they are the same at the precision 0.1 and 0.01 :

\begin{tabular}{|r|r|r|r|}
\hline \multicolumn{2}{|c|}{$\alpha=0.1$} & \multicolumn{2}{c|}{$\alpha=0.01$} \\
\hline$X_{1}^{\alpha}$ & $X_{4}^{\alpha}$ & $X_{1}^{\alpha}$ & $X_{4}^{\alpha}$ \\
\hline$-0.3109-0.6469 \mathrm{i}$ & $-0.1737-0.6105 \mathrm{i}$ & $-0.3505-0.6612 \mathrm{i}$ & $-0.3374-0.6562 \mathrm{i}$ \\
$1.1055+1.1782 \mathrm{i}$ & $1.1135+1.3751 \mathrm{i}$ & $1.0799+1.1021 \mathrm{i}$ & $1.0896+1.1283 \mathrm{i}$ \\
$0.5715-0.3795 \mathrm{i}$ & $0.6686-0.0867 \mathrm{i}$ & $0.5338-0.4673 \mathrm{i}$ & $0.5468-0.4382 \mathrm{i}$ \\
$-0.3463+0.9663 \mathrm{i}$ & $-0.1694+1.0052 \mathrm{i}$ & $-0.3969+0.9582 \mathrm{i}$ & $-0.3802+0.9607 \mathrm{i}$ \\
$0.4859+0.2819 \mathrm{i}$ & $0.2775+0.5302 \mathrm{i}$ & $0.5458+0.2025 \mathrm{i}$ & $0.5261+0.2291 \mathrm{i}$ \\
$0.6330-0.9571 \mathrm{i}$ & $0.5969-0.8712 \mathrm{i}$ & $0.6345-0.9699 \mathrm{i}$ & $0.6345-0.9663 \mathrm{i}$ \\
$-0.9443-0.3735 \mathrm{i}$ & $-0.9402-0.4181 \mathrm{i}$ & $-0.9396-0.3575 \mathrm{i}$ & $-0.9414-0.3630 \mathrm{i}$ \\
$-1.1099+0.6015 \mathrm{i}$ & $-1.0772+0.4499 \mathrm{i}$ & $-1.1081+0.6460 \mathrm{i}$ & $-1.1093+0.6313 \mathrm{i}$ \\
$0.3053-0.0158 \mathrm{i}$ & $0.0636+0.2721 \mathrm{i}$ & $0.3643-0.0974 \mathrm{i}$ & $0.3454-0.0706 \mathrm{i}$ \\
\hline$\alpha=0.001$ & $X_{4}^{\alpha}$ & \multicolumn{2}{|c|}{$\alpha=0.0001$} \\
\hline \multicolumn{2}{|c|}{$X_{1}^{\alpha}$} & $X_{4}^{\alpha}$ \\
\hline$-0.3544-0.6627 \mathrm{i}$ & $-0.3531-0.6622 \mathrm{i}$ & $-0.3548-0.6628 \mathrm{i}$ & $-0.3546-0.6628 \mathrm{i}$ \\
$1.0767+1.0942 \mathrm{i}$ & $1.0778+1.0968 \mathrm{i}$ & $1.0764+1.0934 \mathrm{i}$ & $1.0765+1.0936 \mathrm{i}$ \\
$0.5298-0.4761 \mathrm{i}$ & $0.5312-0.4731 \mathrm{i}$ & $0.5294-0.4769 \mathrm{i}$ & $0.5296-0.4766 \mathrm{i}$ \\
$-0.4018+0.9575 \mathrm{i}$ & $-0.4002+0.9577 \mathrm{i}$ & $-0.4023+0.9574 \mathrm{i}$ & $-0.4022+0.9575 \mathrm{i}$ \\
$0.5517+0.1945 \mathrm{i}$ & $0.5497+0.1972 \mathrm{i}$ & $0.5523+0.1937 \mathrm{i}$ & $0.5521+0.1940 \mathrm{i}$ \\
$0.6344-0.9708 \mathrm{i}$ & $0.6344-0.9705 \mathrm{i}$ & $0.6344-0.9709 \mathrm{i}$ & $0.6344-0.9709 \mathrm{i}$ \\
$-0.9389-0.3559 \mathrm{i}$ & $-0.9391-0.3564 \mathrm{i}$ & $-0.9389-0.3557 \mathrm{i}$ & $-0.9389-0.3558 \mathrm{i}$ \\
$-1.1077+0.6504 \mathrm{i}$ & $-1.1078+0.6489 \mathrm{i}$ & $-1.1076+0.6508 \mathrm{i}$ & $-1.1076+0.6507 \mathrm{i}$ \\
$0.3698-0.1054 \mathrm{i}$ & $0.3679-0.1028 \mathrm{i}$ & $0.3703-0.1062 \mathrm{i}$ & $0.3701-0.1060 \mathrm{i}$ \\
\hline
\end{tabular}

\begin{tabular}{|r|}
\hline \multicolumn{1}{|c|}{$X_{1}=X_{4}$} \\
\hline$-0.3548-0.7822 \mathrm{i}$ \\
$1.0764+0.0441 \mathrm{i}$ \\
$0.5294-0.2717 \mathrm{i}$ \\
$-0.4024-0.8097 \mathrm{i}$ \\
$0.5523-0.2585 \mathrm{i}$ \\
$0.6344-0.2111 \mathrm{i}$ \\
$-0.9389-1.1194 \mathrm{i}$ \\
$-1.1076-1.2168 \mathrm{i}$ \\
$0.3704-0.3635 \mathrm{i}$ \\
\hline
\end{tabular}

For each $n$, The sequence $\left(X_{n}^{\alpha}\right)_{\alpha}$ clearly converges towards $X_{n}$, when $\alpha \rightarrow 0$. 


\section{Acknowledgements}

The authors wish to thank the editor and the anonymous referees for their valuable and helpful comments and suggestions which improved the initial version of this paper. We also wish to thank the STAPH group in Toulouse (http://www.math.univ-toulouse.fr/staph/index.html) for stimulating discussions.

\section{References}

Azencott, R. and Dacunha-Castelle, D. (1984). Séries d'observations irrégulières. Masson, Paris

Bosq, D. (2000). Linear Processes in Function Spaces. Theory and Applications. Lecture notes in statistics, 149, Springer, Berlin.

Boudou, A. (2007). Groupe d'opérateurs unitaires déduit d'une mesure spectrale - une application. C. R. Acad. Sci. Paris, Ser. I 344 791-794

Boudou, A. and Romain, Y. (2002). On spectral and random measures associated to continuous and discrete time processes. Stat. Proba. Letters 59 145-157.

Brillinger, D. R. (2001). Time Series: Data Analysis and Theory. 2nd ed. Society for Industrial Applied Mathematics, Philadelphia.

Dehay, D. and Monsan, V. (2007) Discrete Periodic Sampling with Jitter and Almost Periodically Correlated Processes. Stat. Infer. Stoch. Process. 10 223-253.

Kato, T. (1980). Perturbation Theory for Linear Operators. Springer-Verlag.

Riesz, F. and Nagy, B. (1991). Functional Analysis. Dover Publications.

Rozanov, Yu. A. (1967). Stationary Random Processes. Holden-Day, Inc, San Francisco.

Shumway, R. H. and Stoffer, Da. S. (2006). Stationary Time Series Analysis and its Applications. Springer, New-York. 